$\mathrm{AB} 1319$

EFFICACY AND SAFETY OF THE TREATMENT WITH JAK INHIBITORS IN PATIENTS WITH RA. RESULTS OF A CASE SERIES OF USUAL CLINICAL PRACTICE

Blanca Hernández-Cruz, Dolores Ruiz-Montesinos, José Javier Pérez Venegas. hospital universitario virgen macarena, Rheumatology, Seville, Spain

Background: The JAK inhibitors, a new group of targeted synthetic DMARDs were marketed in Spain in 2018. These oral drugs are a good therapeutic alternative for patients with MTX failure. These are not biologic DMARDs and its place in clinical practice is not clear.

Objectives: To identify the clinical characteristics of the patients treated with JAK inhibitors in a Rheumatology service in the South of Spain. To know the efficacy and safety of these drugs used in usual clinical practice.

Methods: Setting: Rheumatology department in a 3rd level University Hospital.

Design: an observational and analytical case series.

From September 2018 to January 15, 2019 clinical data were collected of all patients with RA (ACR/EULAR 2010) treated with JAK inhibitors. The variables collected were demographics, clinical, related to RA, comorbidities, activity (DAS28ESR) and EULAR response. Safety variables included severe adverse events, and adverse events that caused the interruption of the treatment. The protocol was approved and conducted according to the local ethic committee regulations. The statistical analysis was done with descriptive and inferential non parametric tests. Data are shown as median (percentile 25 - percentile 75).

Results: In the study period, 33 patients were treated with JAK inhibitors. Two patients were excluded of this review due to lack of an evaluation of follow-up. Data of $31 \mathrm{RA}$ patients are presented. They were female (74\%) with RF+, ACPA+ and erosive disease en more of $90 \%$ of cases; $61 \%$ had at least one comorbidity. The Charlson comorbidity index was $3(2-6)$. The median age at the time of the start of the JAK inhibitor was $63.3(56.8$ - 70.7) years. The disease duration at the time of the start of the JAK inhibitor was $14.7(8.6-27.5)$ years. All the patients had received synthetic conventional DMARDs, the number of previous scDMARDs was $2(2-6) ; 26 \%$ had been treated with biologic DMARDs. The number of previous bDMARDS was $2(1-5) ; 26(84 \%)$ patients were treated with baricitinib and 5 with tofacitinib, oh these 25 $(77 \%)$ were treated with corticosteroids, the median daily dose of prednisone was $7.5(5-15) \mathrm{mg} / \mathrm{day}$, and $16(52 \%)$ were treated with MTX, the mean weekly dose was $15(10-25) \mathrm{mg} / \mathrm{week}$. The baseline DAS28ESR was $4.9(4.1-5.7)$ and the end DAS28ESR was of 2.6 (2.07 - 3.5), $p<0.0001$; the EULAR response was good in $15(52 \%)$, Moderate in $11(35 \%)$ and Poor in 5 (13\%). At the last visit $15(38 \%)$ were in remission (DAS28ESR $\leq 2.6$ ), and the follow-up was of 11.1 (6.8 - 16.7) months. In 8 patients the treatment was stopped, one due to primary inefficacy, three due to secondary inefficacy and in five patients due to adverse events The most relevant adverse events were one case with septic arthritis of the knee after a intra articular injection; one case with a community acquired pneumonia; one case with pyometra, one breast cancer recurrence, one severe neutropenia and one with severe psoriasis flare. There were no thrombotic events. The only variable related with remission was the low baseline DAS.

Conclusion: The JAK inhibitors are tsDMARD with good profile of efficacy and safety in clinical practice. The patients treated with these drugs had a long and severe disease with failure to several drugs and poor prognostic variables, and a high comorbidity index, however these drugs show good response rates.

Disclosure of Interests: None declared

DOI: 10.1136/annrheumdis-2019-eular.4436

\section{AB1320 NEUTROPHIL-TO-LYMPHOCYTE RATIO AND PLATELET- TO-LYMPHOCYTE RATIO ARE ASSOCIATED WITH DISEASE ACTIVITY IN POLYMYALGIA RHEUMATICA}

Ju-Yang Jung, Jiwon Kim, Chang-Hee Suh, Hyoun-Ah Kim. Ajou University School of Medicine, Rheumatology, Suwon, Korea, Rep. of (South Korea)

Background: Polymyalgia rheumatica (PMR) is a systemic inflammatory disorder, which develops inflammatory pain and stiffness of large joints. There is no specific serological marker or test to differentiate PMR.

Objectives: In this study, we compared the neutrophil-to-lymphocyte ratio (NLR), platelet-to-lymphocyte ratio (PLR), and monocyte-to-lymphocyte ratio (MLR) among patients with polymyalgia rheumatica (PMR) and rheumatoid arthritis (RA), and explored possible associations with clinical features, disease activity, and prognosis in PMR.
Methods: A total of 94 PMR and 242 RA patients who were initially diagnosed at the rheumatology clinic of a university-based tertiary hospital were enrolled. Symptoms, physical examination, and medical histories were collected with the results of laboratory tests.

Results: NLR $(4.5 \pm 3.3$ vs. $2.8 \pm 1.8)$, PLR $(222.7 \pm 115.5$ vs. $159.7 \pm$ $78.1)$, and MLR $(0.4 \pm 0.3$ vs. $0.3 \pm 0.2)$ were higher in patients with PMR compared with patients with RA (all $p<0.001$ ). NLR, PLR, and MLR were correlated with specific serological values, including CRP and albumin, in patients with PMR. After disease activity resolved, NLR $(2.95$ $\pm 2.32, \mathrm{p}<0.001)$, PLR $(137.5 \pm 82.3, \mathrm{p}<0.001)$, and MLR $(0.26 \pm$ $0.16, p<0.001)$ decreased significantly. By comparing patients according to the disease course, swollen joint counts were higher in the chronic course group compared with the remission group $(p=0.03)$, while NLR, PLR, and MLR levels were similar.

Conclusion: This study found that NLR, PLR, and MLR in patients with PMR were higher than in those with RA. Furthermore, NLR, PLR, and MLR levels were associated with disease activity and specific clinical features, although they could not predict prognosis in patients with PMR.

\section{REFERENCES:}

[1] van der Geest KS, Abdulahad WH, Rutgers A, Horst G, Bijzet J, Arends $\mathrm{S}$, et al. Serum markers associated with disease activity in giant cell arteritis and polymyalgia rheumatica. Rheumatology (Oxford). 2015;54(8):1397402.

[2] Erre GL, Paliogiannis P, Castagna F, Mangoni AA, Carru C, Passiu G, et al. Meta-analysis of neutrophil-to-lymphocyte and platelet-to-lymphocyte ratio in rheumatoid arthritis. Eur J Clin Invest. 2018:e13037

Disclosure of Interests: Ju-Yang Jung: None declared, Jiwon Kim: None declared, Chang-Hee Suh Consultant for: Celltrion, Inc, Hyoun-Ah Kim: None declared

DOI: 10.1136/annrheumdis-2019-eular.5471

\section{AB1321 PSYCHOMETRIC PROPERTIES OF THE ASAS HEALTH INDEX IN PATIENTS WITH ACTIVE AS/RADIOGRAPHIC AXIAL SPA WHO HAVE PRIOR INADEQUATE RESPONSE/INTOLERANCE TO TNF INHIBITORS IN A PHASE 3 TRIAL}

Uta Kiltz ${ }^{1,2}$, Désirée van der Heijde ${ }^{3}$, Annelies Boonen ${ }^{4}$, Lianne S. Gensler ${ }^{5}$, Theresa Hunter ${ }^{6}$, Kathleen Wyrwich ${ }^{6}$, Yan Dong ${ }^{6}$, LI Xiaogi ${ }^{6}$, Gaia Gallo ${ }^{6}$, Juergen Braun ${ }^{1,2} .{ }^{1}$ Rheumazentrum Ruhrgebiet, Herne, Germany; ${ }^{2}$ RuhrUniversität Bochum, Bochum, Germany; ${ }^{3}$ Leiden University Medical Centre, Leiden, Germany; ${ }^{4}$ Maastricht University Medical Center, Maastricht, Netherlands; ${ }^{5}$ UCSF School of Medicine, San Francisco, United States of America; ${ }^{6}$ Eli Lilly and Company, Indianapolis, United States of America

Background: The Assessment of SpondyloArthritis international Society Health Index (ASAS HI) assesses function, disability, and health in patients with ankylosing spondylitis/radiographic axial spondyloarthritis (AS/ r-axSpA).

Objectives: To evaluate the psychometric properties of the ASAS $\mathrm{HI}$ in patients with active AS/r-axSpA in a placebo-controlled study of ixekizumab, a humanized IL-17A monoclonal antibody (COAST-W, NCT02696798).

Methods: The ASAS HI questionnaire consists of 17 dichotomous items that yield a total score ranging from 0 (good health) to 17 (poor health). The psychometric properties of the questionnaire, including test-retest reliability, convergent and discriminant validity, and responsiveness, were evaluated using pooled data from three exposure groups (placebo and ixekizumab $80 \mathrm{mg}$ every 2 or 4 weeks). Adults enrolled fulfilled ASAS criteria (sacroilitis defined centrally by modified New York Criteria and $\geq 1$ SpA feature), had active disease (BASDAI $>4$, back pain $>4$ ), and either prior inadequate response or intolerance to 1 or 2 TNF inhibitors.

Results: Mean baseline ASAS HI score was 9.7 ( $\mathrm{SD}=3.62, \mathrm{n}=316)$. Intraclass correlation (0.78) indicated test-retest reliability of ASAS HI between screening and baseline. ASAS $\mathrm{HI}$ score was moderately correlated at baseline $(|\mathrm{r}|>0.30$, Figure [circles]) and strongly correlated at Week (Wk) $16(|r|>0.50$; Figure [crosses]) with BASDAl, BASFI, SF-36 PCS, and spinal pain, and strongly correlated at both baseline and Wk 16 with EQ5D-5L UK Population-based Index and SF-36 MCS. Greater improvements in disease activity were associated with greater improvements in ASAS $\mathrm{HI}$ scores at Wk 16 (Table).

Conclusion: The ASAS HI demonstrated reliability, validity, and responsiveness in adults with $\mathrm{AS} / \mathrm{r}$-axSpA, supporting its use in clinical trials. 